\title{
Breathing mode in the Bose-Hubbard chain with a harmonic trapping potential
}

\author{
Wladimir Tschischik, Roderich Moessner, and Masudul Haque \\ Max-Planck-Institut für Physik komplexer Systeme, Nöthnitzer Str. 38, $0118^{77}$ Dresden, Germany
}

(Dated: November 9, 2018)

\begin{abstract}
We investigate the breathing mode of harmonically trapped bosons in an optical lattice at small site occupancies. The Bose-Hubbard model with a trapping potential is used to describe the breathing-mode dynamics initiated through weak quenches of the trap strength. We connect to results for continuum bosons (Lieb-Liniger and Gross-Pitaevskii results) and also present deviations from continuum physics. We take a spectral perspective, identifying the breathing mode frequency with a particular energy gap in the spectrum of the trapped Bose-Hubbard Hamiltonian. We present the low energy eigenspectrum of the trapped many-boson system, and study overlaps of the initial state with eigenstates of the quenched Hamiltonian. There is an intermediate interaction regime, between a "free-boson" limit and a "free-fermion" limit, in which the Bose-Hubbard breathing mode frequency approaches the Gross-Pitaevskii prediction. In addition, we present a striking failure of the time-dependent Gutzwiller approximation for describing breathing modes.
\end{abstract}

\section{INTRODUCTION}

Controlled and tunable experimental realizations of confined quantum systems with ultra-cold atoms enables non-equilibrium studies of quantum many-body states in novel geometries. A common feature of many cold-atom experiments is the confinement of a many-body system in a harmonic trap. Trapping introduces many distinctive features which have no analog in uniform many-body states, such as collective excitations like breathing modes, dipole modes, and scissors modes. Such trap-related collective modes have been widely studied both experimentally and theoretically for continuum systems, especially in the mean-field regime, since the early days after quantum degeneracy was achieved with trapped atoms [1]. In a well-known recent experiment 2, breathing modes have been used for a one-dimensional (1D) system of continuum bosons to characterize mean-field and non-meanfield regimes, as well as states obtained by quenching to large negative interactions. With the addition of optical lattices, it should be possible to study collective modes in lattice systems beyond the mean field regime. In this work, we study the breathing mode for interacting bosons on a 1D lattice, described by the Bose-Hubbard model and subject to an additional trapping potential.

Our work addresses the dynamics of a finite number $(N)$ of bosons on a 1D chain with $L>N$ sites, subject to the Hamiltonian

$$
\begin{aligned}
H_{B H}=- & J \sum_{i=1}^{L-1}\left(b_{i}^{\dagger} b_{i+1}+b_{i+1}^{\dagger} b_{i}\right) \\
& +\frac{U}{2} \sum_{i=1}^{L} \hat{n}_{i}\left(\hat{n}_{i}-1\right)+\sum_{i=1}^{L} V(i) \hat{n}_{i} .
\end{aligned}
$$

Here $b_{i}, b_{i}^{\dagger}$, are the bosonic operators for the site $i$ $(i=1 \ldots L)$, and $\hat{n}_{i}=b_{i}^{\dagger} b_{i}$. We will measure energy [time] in units of the tunnel coupling $J$ [inverse tunnel coupling $1 / J]$, and therefore set $J=\hbar=1$. The trap-

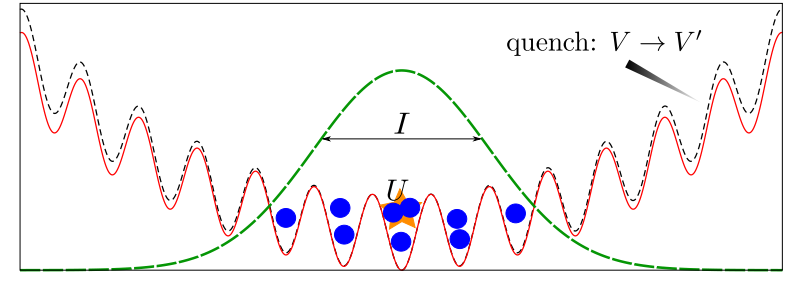

FIG. 1. (color online) Schematic of bosons in an optical lattice and overall harmonic trapping potential. The trap strength is quenched from $V$ to $V^{\prime}$. The bell-shaped (dashed green) curve represents the density profile, shown schematically as a continuous curve; in our tight-binding description it is a discrete function (site occupancies).

ping potential

$$
V(i)=\frac{1}{2} k\left(i-\frac{L+1}{2}\right)^{2}
$$

is centered at the midpoint of the chain.

The breathing mode can be excited by a quench (sudden change) of the trapping potential strength $k$. Fig. 1 shows schematically lattice bosons trapped in a harmonic trap and a quench of the trapping strength $k$, i.e. a reduction of harmonic confinement. In subsequent time evolution the bosonic cloud undergoes expansion and contraction dynamics. We characterize this dynamics using the second moment of the density distribution $I=\sum_{i} \hat{n}_{i}\left(i-\frac{L+1}{2}\right)^{2}$, which measures the cloud width. After a quench the second moment shows oscillatory behavior. If the dynamics $I(t)$ is nearly monochromatic, the dominant frequency $\Omega$ can be identified as the breathing mode frequency. The dynamics is expected to be nearly monochromatic if the quench is small; we will concentrate on small quenches of $k$.

Collective modes in 1D Bose-Hubbard systems have been previously addressed in Refs. 36 6]. We focus on the connection to and deviations from continuum physics, and hence concentrate on situations where the site occupancy is everywhere lower than unity. This precludes 
Mott physics from the present study. When the density is low enough, we can approximate the cosine dispersion of a lattice particle by a quadratic dispersion; this "effective mass approximation" $m^{*}=\frac{1}{2 J}$ ascribes a continuum mass to lattice particles, so that we can relate our trapping strength $k$ to the trapping frequency of a continuum trapping potential $\frac{1}{2} m \omega_{0}^{2} x^{2}$ :

$$
\omega_{0}=\sqrt{2 k J} .
$$

We will take this to be the definition of the trapping frequency $\omega_{0}$ on the lattice, even when the densities are not small enough for the effective mass approximation to hold.

We will compare with results and approximations from the continuum case. The continuum limit of the BoseHubbard model is the Lieb-Liniger model

$$
\begin{aligned}
H_{L L}=-\frac{\hbar^{2}}{2 m} \sum_{j=1}^{N} \frac{\partial^{2}}{\partial x_{j}^{2}}+c \sum_{\langle i, j\rangle} & \delta\left(x_{i}-x_{j}\right) \\
+ & \frac{1}{2} m \omega_{0}^{2} \sum_{i} x_{i}^{2},
\end{aligned}
$$

where we have added a trap term. Full quantum calculations of Lieb-Liniger dynamics in harmonic traps may be possible using the methods of Refs. [7, where related situations have been studied. Refs. 8 11] have recently used Multi-Configuration Time-Dependent Hartree approaches to calculate breathing dynamics and/or eigenspectra of harmonically trapped continuum bosons in a trap; the lattice results of the present work complement these continuum results. The breathing mode of harmonic-trapped 1D continuum bosons has also been addressed through hydrodynamic ideas, kinetic equations, lattice approximations, and sum rules [6, 12 17]. The breathing mode for trapped 1D continuum bosons has been experimentally measured [2, 18].

When bosons form a Bose-Einstein condensate, the dynamics of the condensate is well-described in the continuum through the time-dependent Gross-Pitaevskii (GP) equation

$$
i \partial_{t} \Psi(r, t)=\left(-\frac{\hbar^{2}}{2 m} \Delta+V(r)+g|\Psi(r, t)|^{2}\right) \Psi(r, t) .
$$

The GP or 'hydrodynamic' description predicts breathing mode frequencies $\Omega_{G P}=\omega_{0} \sqrt{D+2}$, for $D$ dimensions [19], i.e., $\Omega_{G P}=\sqrt{3} \omega_{0}$ for $1 \mathrm{D}$, with weak dependence on the interaction parameter $g$.

The Gross-Pitaevskii description is reliable for higher dimensions, small temperatures, and weak interactions. Since there is no true condensation for 1D bosons, one might wonder whether the prediction has any relevance to the 1D system we are studying. The experiment of Ref. 2] has found a regime of interactions where the breathing-mode frequency approaches the GrossPitaevskii prediction. The lattice calculations presented in this article, and the continuum calculations of Ref. [10, show that, even for true 1D bosons having no transverse degrees of freedom whatsoever, there is a range of interactions where the breathing frequency approaches $\sqrt{3} \omega_{0}$.

We will pay particular attention to the spectrum. Since the many-body system in a trap is a paradigmatic situation in cold-atom experiments, a thorough description of many-body eigenspectra in the presence of traps is clearly of fundamental interest 8 11, 20]. We provide a description of the eigenspectra of 1D many-boson systems in harmonic traps and relate spectral properties to the breathing mode excitation. This description applies qualitatively both to the 1D Bose-Hubbard model as a function of the on-site interaction, and to the LiebLiniger model as a function of the contact interaction.

The breathing mode frequency can be identified as the excitation energy of the lowest spatially symmetric manybody eigenmode that is excited in a trap quench. For small enough quenches, we can neglect the occupation of all but one of the excited states. If the ground state and this excited state have energies $E_{0}$ and $E_{n}$, the wavefunction evolves as $|\psi(t)\rangle=a_{0} e^{-i E_{0} t}\left|\phi_{0}\right\rangle+a_{n} e^{-i E_{n} t}\left|\phi_{n}\right\rangle$. As a result, any observable, including the cloud size, will have oscillation frequency $\Omega=E_{n}-E_{0}$. In Sections II and III we will identify the energy level relevant for a small trap quench; we provide a brief summary here.

For non-interacting trapped bosons $(U=0)$ in the continuum, the first excited state is at energy $\hbar \omega_{0}$ and spatially asymmetric. There are two degenerate, spatially symmetric, states at energy $2 \hbar \omega_{0}$. The same situation occurs in the $U \rightarrow \infty$ limit where the bosons act as free fermions (Tonks-Girardeau limit). One of these degenerate levels stays flat at $2 \hbar \omega_{0}$ for all $U$. In a spatially symmetric trap quench, however, it is the other level of this pair that gets dominantly excited. The breathing mode frequency as a function of $U$ can be identified with the excitation energy of this level, which goes to $2 \omega_{0}$ in the limits $U=0, \infty$. At finite $U$, we show that this frequency (energy level) drops below $2 \omega_{0}$, and the minimum value approaches $\sqrt{3} \omega_{0}$ as the particle number is increased. Of course, this description is strictly valid only in the continuum; for the Bose-Hubbard model there are deviations at finite densities.

In addition to the exact calculations for spectra and breathing frequencies, we have employed the time-dependent Gutzwiller approximation for the BoseHubbard model. Despite the popularity of this timedependent mean-field approximation, regimes where this might give incorrect dynamics are not widely known. We have found that this approximation gives qualitatively incorrect results for the breathing frequency at large interactions.

The spectrum and the breathing mode frequency, as functions of particle number and density, are described in Sections [I] and III. Section IV]describes the Gutzwiller approximation and its failure to describe breathing mode frequencies at large $U$. In Section $\mathrm{V}$ we present some 


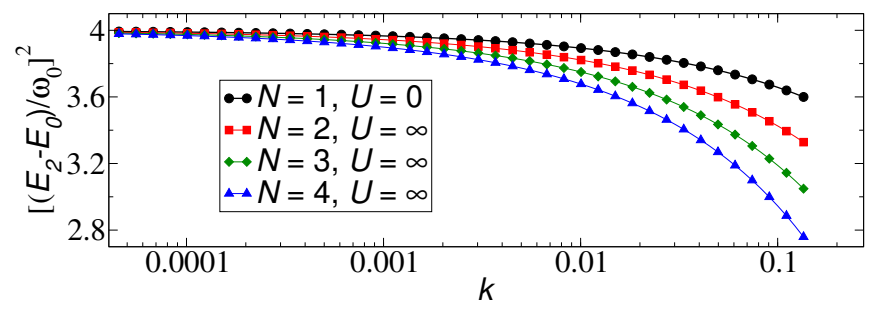

FIG. 2. The second excitation energy (breathing mode frequency, $\left.E_{2}-E_{0}\right)$ at the free-boson $(U=0)$ and free-fermion $(U=\infty)$ points. The excitation energy is equal to $2 \omega_{0}$ in the continuum limit (small $k$ ) and deviates significantly for stronger traps.

features of strong traps, farther from the regime where the continuum approximation is applicable.

\section{SPECTRUM OF A BOSE-HUBBARD CHAIN WITH A HARMONIC TRAP}

We describe below the many-boson spectrum for a Bose-Hubbard chain at low filling in the presence of a harmonic trap, both for the integrable points $(U=0$ and $U=\infty$, subsection II A) and for general $U$ (subsection II B. We first describe continuum expectations and then show how the finite-density lattice system deviates. The continuum case (1D trapped bosons) has been discussed in the literature previously $9,11,20$.

\section{A. $U=0$ (free bosons) and $U=\infty$ ("free fermions")}

In the limits $U=0$ (non-interacting bosons) and $U=$ $\infty$ (hard-core bosons, Tonks-Girardeau limit), the manybody spectrum can be constructed out of the singleparticle spectrum.

In the continuum, the single-particle spectrum is the equally spaced harmonic oscillator spectrum. The free bosonic ground state is then constructed by placing all bosons in the single-particle ground state, and excited states are constructed by promoting bosons to higher single-particle states. For large $N$, the number of ways one can distribute the $N$ particles in single-particle levels to get a many-body excited state of excitation energy $E_{i}-E_{0}=l \omega_{0}$ is the number of integer partitions $p(l)$ of the integer $l[21$. For finite $N$, the degeneracy sequence of the many-body spectrum is modified to the sequence $p_{N}(l)$ given by the number of partitions of $l$ into a maximum of $N$ parts 22 .

The free fermion (hard-core boson) ground state is constructed by filling up the lowest $N$ single-particle states. Therefore, in the continuum the free fermion ground state energy is larger than the free-boson ground state energy by $\frac{1}{2} N(N-1) \omega_{0}$. The degeneracy counting of excitations over this ground state is the same as in the free-boson case.

In the presence of a lattice, the single-particle spectrum is modified 4 and no longer equally spaced. Figure 2 shows the excitation energy of the second excited level for lattice bosons. For $U=0$, the excitation energy is the same for any number of bosons, and is equal to the excitation energy of the second single-particle state. (The many-body excited state is formed by promoting a single particle from the condensate to this excited level.) For $U=\infty$, the many-body excited eigenstate is constructed by promoting a particle across the "Fermi surface"; hence the second many-body excitation energy is the difference between the $N$-th and $(N+2)$-th single-particle level, and thus depends on the number of particles. Figure 2 shows that the deviation of $E_{2}-E_{0}$ from the continuum value $2 \omega_{0}$ is smallest for weak traps, when the central occupancy is low, and increases with increasing $k$. For large $k$, the bosonic cloud is localized on few lattice cites. Such situations are not closely related to continuum physics and are described in Section $\mathrm{V}$.

For low densities, the $U=0$ ground state is expected to be $\sim N \frac{1}{2} \omega_{0}$ above the bottom of the band, which is at energy $-2 J N$. This is seen to be approximately true for $N=3$ bosons and $\omega_{0}=\sqrt{2 k} \approx 0.0447$ in Figure 3 a. In the same figure we also see the difference between the bosonic and fermionic ground states to be approximately $\frac{1}{2} N(N-1) \omega_{0}$.

For finite chains, the additional confinement of the chain edges also affects the spectrum. The effect is stronger for higher excited states and large $U$ because the relevant eigenstates are spatially more extended. As seen in Figure $3 \mathrm{~b}$, the effect is to increase the excitation energies.

\section{B. Finite $U$}

We first describe the spectrum in the continuum.

The ground state energy increases monotonically with $U$, changing by $\frac{1}{2} N(N-1) \omega_{0}$ as $U$ is varied from $U=0$ to $U=\infty$. Since we are interested in frequencies observed in real-time dynamics, we will focus on the excitation energies.

A prominent feature of the excitation spectrum is that, at each $l \omega_{0}$, there is a single energy level that remains constant as a function of $U$. These are related to the dipole oscillation (Kohn) mode [24. Introducing centerof-mass and relative coordinates it is possible to show that the inter-particle interaction appears only in the Hamiltonian describing the relative motion sector. Since the center of mass dynamics of the system is independent of the interaction, an equally spaced harmonic oscillator spectrum appears within the full many-body spectrum at any $U$. Formally, there exists a collective ladder operator, commuting with the interaction term [24], which creates a tower of equally spaced eigenenergies separated by $\omega_{0}$, independently of the interaction.

At each $l \omega_{0}$ with $l>1$, the eigenenergies other than the 

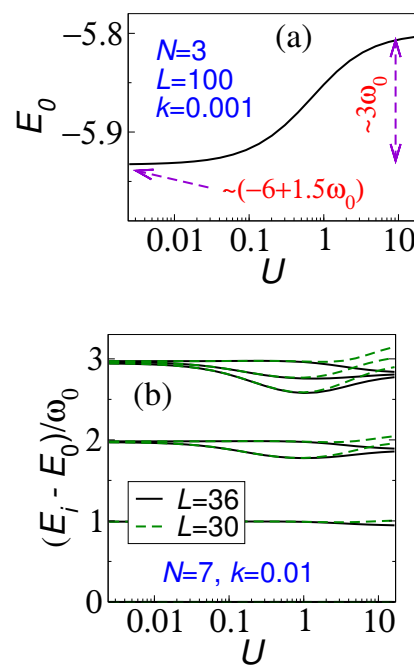

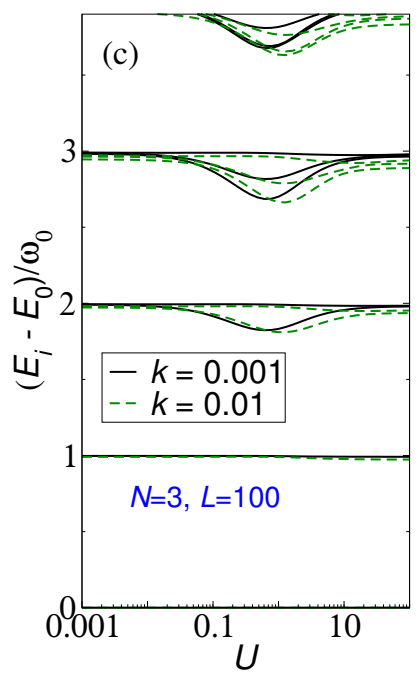

FIG. 3. Low-energy spectrum of the 1D Bose-Hubbard model with a trap. (a) Ground state energies for $N=3$ bosons with weak trapping. (b) $N=7$ bosons; comparison of two chain lengths shows the effect of finite edges on the excitation energies. (c) $N=3$ bosons; comparison of two trapping potentials shows the deviation from continuum with stronger traps.

flat level vary non-monotonically with $U$, dipping below $l \omega_{0}$ at some finite $U$. We will associate the lowest of these non-constant excitation energies with the breathing mode frequency.

Figure $3 \mathrm{~b}, \mathrm{c}$ displays this structure for the lowest few levels. Since our calculations are on a lattice, there are (small) deviations from the continuum case, which are characterized in Figure 3. by comparing two different trap strengths. (Smaller $k$ corresponds more closely to the continuum.) For a lattice system, the exact degeneracies at $U=0$ and $U=\infty$ are spoiled, and the levels related to the dipole mode are not completely independent of $U$. Lattice effects push the energies below their continuum values.

\section{BREATHING MODE FREQUENCY}

In this section we show the connection of the breathing mode frequency $\Omega$ to the low energy spectrum, by analyzing the overlaps of the initial state with the final eigenstates in quenches of the trap strength (III B). We then describe the dependence of the breathing mode frequency on the interaction strength $U$ (IIIC).

\section{A. Quench dynamics}

We excite breathing modes through trap quenches. The initial state is the ground state of an initial Hamiltonian $H\left(k_{i}\right)$ with trap strength $k_{i}$. At time $t=0$ the trapping strength is suddenly reduced to $k<k_{i}$; the

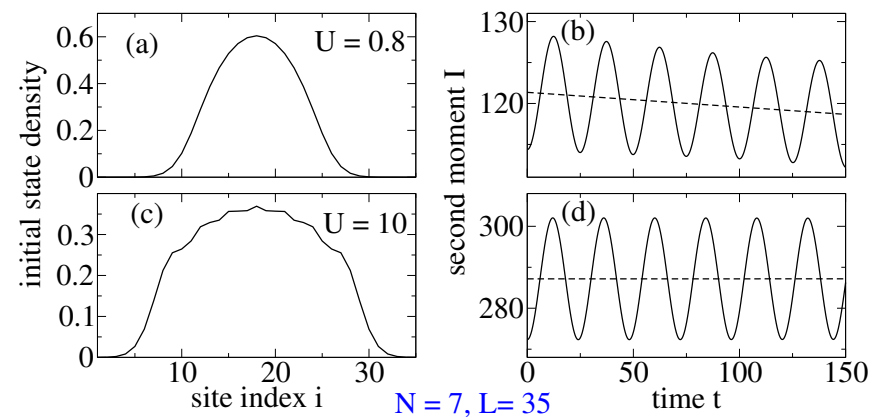

FIG. 4. (a,c) Ground state density profiles for $N=7$ bosons on $L=35$ lattice sites at $k=0.011$ and (a) $U=0.8$ and (c) $U=10$. For small $U$ density distribution is Gaussian-like; for large $U$ the density distribution shows oscillations (fermionization). (b,d) Breathing mode oscillation after a quench of trapping strength $k=0.011 \rightarrow 0.01$. (b) For $U=0.8$ the influence of a second frequency is seen through the overall decrease in addition to oscillations. (d) For $U=10$ there is no noticeable influence of additional frequencies.

trapped bosonic cloud then undergoes breathing mode oscillations. We perform small quenches, reducing the trapping strength by $5 \%$ to $10 \%$.

Fig. 4(a,c) show the initial density distributions for $N=7$ bosons with $k=0.011$ and $U=0.8(U=10)$. The density profiles are near-Gaussian for small interactions. At large $U$ fermionization is discernible as peaks (oscillations) of the density distribution [23.

In Figs. 4(b,d) we show the time evolution of the second moment of the cloud after the trap strength is quenched from $k=0.011$ to $k=0.01$. There is a clear dominant frequency of oscillation. We find it natural to identify this dominant frequency as the breathing mode frequency. At large $U$, no other feature is visible at these timescales. At small $U$, however, the envelope of the oscillating $I(t)$ has an overall decrease, indicating that at least two different frequencies are present in the time evolution.

\section{B. Overlaps with eigenstates of the quenched Hamiltonian}

For small quenches the initial state is a superposition of only the lowest eigenstates of the quenched Hamiltonian. The largest overlap (near unity) is with the new ground state. The first eigenstate is not excited because of reflection symmetry (it is odd under spatial parity, as opposed to the initial state). We therefore concentrate on the second and third excited states, as the eigenstates higher than that have very small weight.

In Figure $5(a, b)$ we show the case of two bosons. For $k<10^{-5}$, the occupancies are small and the effective mass approximation works well, so that these can be considered to be continuum results. The overlap with the second excited state is seen to be consistently larger than the overlap with the third excited state, except at 

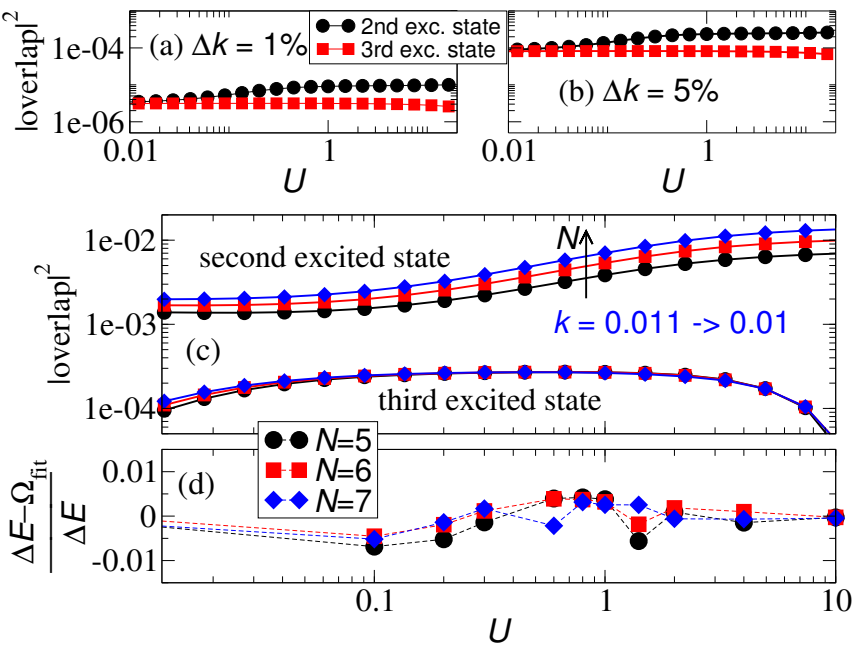

FIG. 5. (color online) (a-c) Overlaps of the initial state with second and third exited states after a quench of trapping strength $k$. (a,b) $N=2$ particles. $1 \%$ and $5 \%$ reductions of $k$ starting from $k=9 \times 10^{-6}$. The separation between 2 nd and 3rd eigenstates at small $U$ is not reliable because of neardegeneracy. (c) Overlaps for $N=5,6,7$. Difference between two overlaps increases with $N$. (d) Relative difference between $\Delta E=E_{2}-E_{0}$ and breathing frequency $\Omega_{f i t}$ obtained by a sinusoidal fitting of the oscillating second moment $I(t)$.

very small $U$. At small $U$, these two excited states are nearly degenerate (c.f., Fig.3), so the relative values of overlaps in this interactions regime does not affect the breathing frequency. Comparison of panels (a) and (b) shows that the relative magnitudes of these two overlaps (second and third excited states) overlaps do not depend strongly of the quench strength, but the absolute magnitudes increase for larger quench strengths, at the expense of the overlap with the new ground state.

The difference between overlaps with the second and third excited states increases with increasing particle number, as shown in Figure 5c for $N=5,6,7$, for the fixed trap quench $k=0.011 \rightarrow 0.01$. With $N=7$ particles, the overlap for the second excited state is larger by more than an order of magnitude than the overlap with the third excited state, over the entire range of interactions.

The overlap results indicate that we can identify the excitation energy of the second excited state as the breathing mode frequency, and that this identification should get better at larger sizes. This is verified by comparing the the excitation energy $E_{2}-E_{0}$ with the frequency of oscillation of the second moment $I(t)$, as shown in Figure 5(d).

The relative magnitudes of the overlaps also explain the $I(t)$ behaviors in Figures 4 $(\mathrm{b}, \mathrm{d})$. At smaller $U$ the third excited state is not completely negligible, and therefore the dynamics, although dominated by the breathing frequency $\Omega=E_{2}-E_{0}$, also contains the frequencies $E_{3}-E_{0}$ and $E_{3}-E_{2}$. The resulting "beating" explains the overall decrease of the oscillating $I(t)$, visible at small

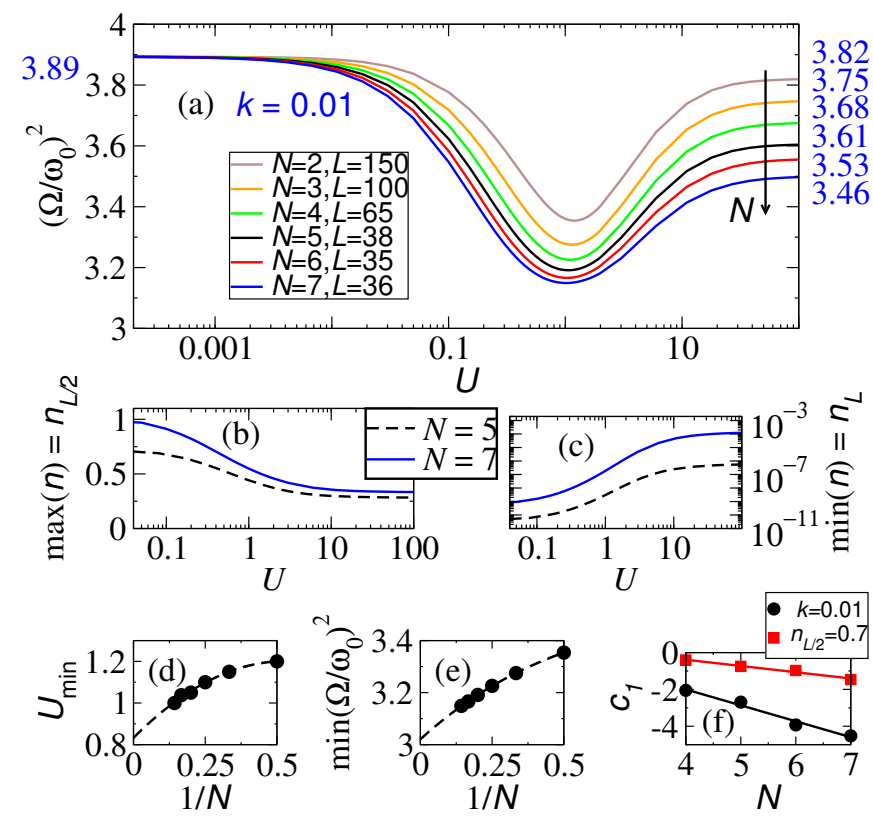

FIG. 6. (color online) (a) Breathing frequency $\Omega$ as a function of interaction $U$, at a fixed trapping strength $k=0.01$. The numbers on the left (right) show $U=0(U=\infty)$ values at large $L$. (b,c) Maximum and minimum of ground state density, for $N=5, L=38$ (full curve) and $N=7, L=35$ (dashed curve), at $k=0.01$. (d) Value of the interaction $U$ at which $\Omega$ has a minimum. (e) Minimum value of $\left(\Omega / \omega_{0}\right)^{2}$. In $(\mathrm{d}, \mathrm{e})$, Dashed line is a third-order polynomial extrapolation to $N \rightarrow \infty$. (f) Slope of $\Omega(U)$ at small $U$, for a constant trapping strength $k=0.01$ and constant central occupancy $n_{L / 2} \approx 0.7$.

$U$ [Figure 4(b)]. At larger $U$ the relative contribution of the third excited state is smaller [Figure $5(a, b, c)]$, which explains why almost no beating is visible in Figure $4(\mathrm{~d})$.

\section{Non-monotonic dependence of breathing frequency on $U$}

Having identified the breathing mode frequency $\Omega$ as the energy difference between the ground and second excited states, $\Omega=E_{2}-E_{0}$, in this subsection we describe the dependence of $\Omega$ on the on-site interaction $U$. As in the continuum case [10, we show that for increasing number of particles $\Omega$ approaches $\sqrt{3} \omega_{0}$, the value predicted by Gross-Pitaevskii theory, for intermediate $U$.

Figure 6 summarizes exact diagonalization results for $N=2, \ldots, 7$, for a fixed trapping strength $k=0.01$. For larger particle numbers, this means significant deviation from the low-density limit where the continuum limit (effective mass approximation) is valid, as seen from the values of the central density in Figure 6 b. At $U \rightarrow 0$, the breathing frequency deviates slightly from the continuum value $\Omega=2 \omega_{0}$ but is the same for all $N$, as explained in Sec. IIA despite the increase of the central density with $N$. In the $U \rightarrow \infty$ limit, the deviation from the contin- 
uum $\Omega=2 \omega_{0}$ is stronger for larger $N$. Since we work with finite chains, there is also some deviation visible at large $U$ from the single-particle predictions with infinite lattices. (The predictions are listed on the right of Figure 6a.) This is because the additional confinement due to a finite lattice affects the spectrum, as noted previously in Figure 3. The effect is noticeable even though the ground-state occupancy of the edge site is less than $10^{-3}$ (Figure 6d). For example, at $U=102$ the breathing frequency is $\left(\Omega / \omega_{0}\right)^{2}=3.50$ for $N=7$ and $L=36$, which is larger than 3.46 , the value calculated from the singleparticle spectrum in an infinite lattice with a $k=0.01$ trap.

Despite these "lattice effects" (finite-density effects) at small and large $U$, the non-monotonic behavior of the $\Omega$ versus $U$ curve (Figure 6a) reflects the overall continuum expectation outlined in the Introduction and explored in Ref. [10. For each $N$, there is a prominent minimum of $\Omega$ at a finite $U$. The minimum value is plotted in Figure 6; the available data strongly indicates that the minimum value of $\Omega$ goes to $\sqrt{3} \omega_{0}$ as $N$ is increased at fixed $k$. The position of the minimum moves to smaller $U$ with increasing number (Figure 6d).

An obvious question is whether at larger $N$ the structure remains a pronounced minimum or whether it becomes a broad plateau at or near $\left(\Omega / \omega_{0}\right)^{2}=3$. Figure 6 : provides a partial answer. We note that the large- $N$ limit may be taken in inequivalent ways on the lattice. In Figure 6a-e, we have kept $k$ constant. It is also reasonable to vary $N$ with constant central density, which requires adjusting $k$ for each $N$ and $U$. Figure 6 shows results for both these schemes. We plot the rate of decrease $c_{1}$ of $\Omega$ with $U$, at small $U$. This is extracted by fitting the $\Omega(U)$ function for $U \lesssim 0.15$ with a third-order polynomial; $c_{1}$ is the linear coefficient of this fit. In both cases (constant $k$ and constant $n_{L / 2}$ ), the slope magnitude increases linearly with the number of bosons $N$. This indicates that, with large enough $N$, the breathing frequency goes down from the non-interacting value $\left(\Omega / \omega_{0}\right)^{2}=4$ toward the mean-field value $\left(\Omega / \omega_{0}\right)^{2}=3$ at very small interactions, so that at larger $N$ the $\Omega(U)$ curve should show a broad valley rather than a sharp minimum. This is consistent with the continuum results of Ref. [10].

\section{FAILURE OF GUTZWILLER APPROXIMATION}

In this section we use the Gutzwiller mean field approximation for the Bose-Hubbard model [25, 26] to calculate the time evolution after a trap quench and hence obtain the breathing mode frequency. We show that the Gutzwiller approximation fails dramatically to reproduce the breathing mode frequency at large $U$.

The Gutzwiller approximation uses the product wave function $|\Psi(t)\rangle=\prod_{i} \sum_{n} f_{n}^{(i)}(t)|i, n\rangle$, where $i$ is the site index and $n$ indicates a single site number state. The time-dependent coefficients $f_{n}^{(i)}(t)$ describe the evolution

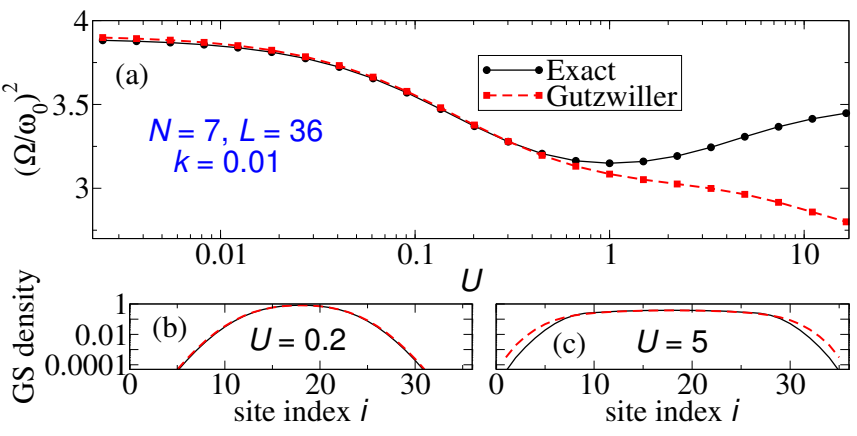

FIG. 7. (color online) (a) Comparison between the exact breathing frequency (obtained from exact diagonalization of the Hamiltonian) and the Gutzwiller approximation (obtained from $I(t)$ oscillations in real-time Gutzwiller evolution). For $U \gtrsim 1$, the Gutzwiller result shows a decrease of $\Omega$, in contradiction to an exact result. (b,c) Ground state density for $k=0.01$, for (b) $U=0.2$ and (c) $U=5$. At large $U$ the ground state density given by the Gutzwiller approximation deviates from the exact result.

of the system. This ansatz captures local number fluctuations but ignores correlations between sites. The dynamics is governed by the coupled differential equations

$$
\begin{aligned}
i \frac{\mathrm{d} f_{n}^{(i)}}{\mathrm{d} t}= & -\sum_{\langle i, j\rangle}\left(\sqrt{n+1} \Phi_{j}^{*} f_{n+1}^{(i)}+\sqrt{n} \Phi_{j} f_{n-1}^{(i)}\right) \\
& +\left(\frac{U}{2} n(n-1)+V(i)\right) f_{n}^{(i)},
\end{aligned}
$$

where $\Phi_{i}=\left\langle b_{i}\right\rangle=\sum_{n} \sqrt{n}\left(f_{n-1}^{(i)}\right)^{*} f_{n}^{(i)}$.

Figure $7 \mathrm{a}$ shows a comparison of the breathing frequency obtained from a trap quench performed using the Gutzwiller approximation, with the exact value calculated from exact diagonalization of the Hamiltonian. For small $U$ the Gutzwiller approximation reproduces well the decrease of $\Omega / \omega_{0}$ from 2 toward $\sqrt{3}$. However, for $U>1$ Gutzwiller shows further decrease of the breathing frequency with increasing $U$.

In Figures 7(b,c) we compare ground states obtained with Gutzwiller theory compared to the exact ground states. There are deviations at large $U$, highlighted through the log-linear scale. Since the same breathing mode frequencies are obtained in different quench strengths, we surmise that the qualitative failure at large $U$ is not due to the initial, but rather due to some fundamental shortcoming of the Gutzwiller approximation which, to the best of our knowledge, is not yet well understood.

One way of viewing this discrepancy is that the Gutzwiller approximation fails to describe the fermionization of the system. However, it is likely that this failure is not a 1D feature only, as the Gutzwiller approximation has been found to incorrectly predict the expansion speed after release from a trap, in both $1 \mathrm{D}$ and higher dimensions 27. 

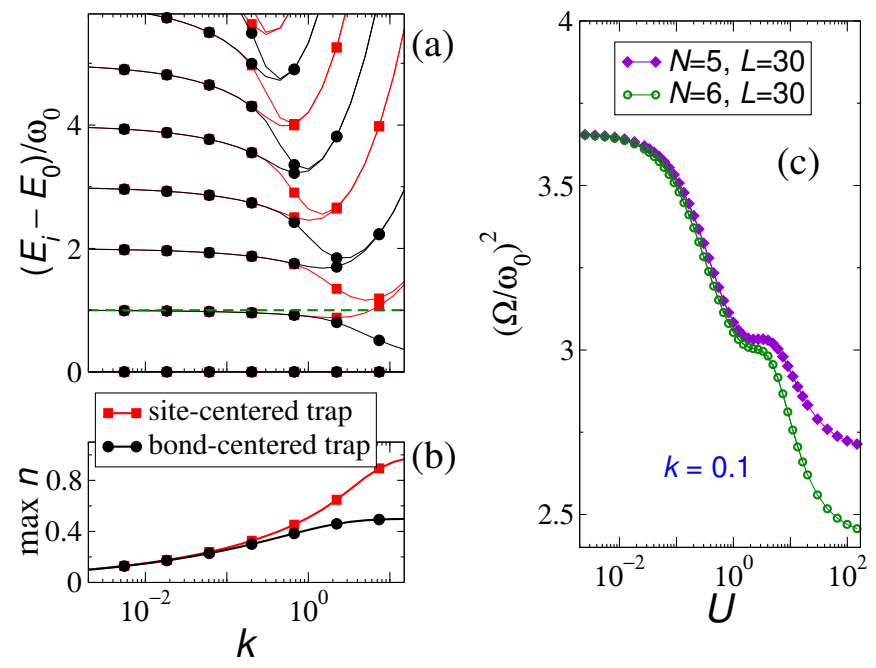

FIG. 8. (color online) (a) Single particle energy spectrum as a function of trapping strength for site-centered trap (oddsized chain) and bond-centered trap (even-sized chain). At larger $k$, the first excited level (dipole mode) deviates from $\omega_{0}=\sqrt{2 k}$, shown as dashed (green) horizontal line. (b) Maximum (central) density of single-particle ground state. (c) Breathing frequency at relatively strong trapping potential $k=0.1$. For $U \gtrsim 1,\left(\Omega / \omega_{0}\right)^{2}$ drops below 3 .

\section{LATTICE EFFECTS AT STRONG CONFINEMENT}

The focus of this work has been on the low-density limit or weak trapping, where comparison to continuum physics is meaningful. In this section we describe some effects of strong trapping situations where the bosons are confined to a few lattice cites.

Figure 8 a shows the single particle spectrum as a function of trapping strength $k$. The energy spectrum gets denser with increasing $k$ as one moves away from the continuum-like limit where the levels are equally spaced. The energy gaps between successive eigenstates decrease with increasing $k$, and this effect is stronger for the higher energy levels. For large $k$ the ground state wavefunction is spread only over a few lattice sites with a high central density (Figure 8b). The deviation from continuum physics, or sensitivity to lattice details, also shows up in the difference between a trap centered at a site (sitecentered, odd lattice) and a trap centered between two sites (bond-centered, even lattice). (In our scheme of placing the trap center at $(L+1) / 2$, this is the difference between odd and even sized lattices.) For $k \rightarrow \infty$ the spectrum is degenerate, except for the ground state in the site-centered case. The failure of the effective mass approximation is also seen through the first excitation en- ergies deviating from $\omega_{0}=\sqrt{2 k}$ as $k$ is increased (Figure 8a).

For larger $k$, as a function of the interaction strength $U$, the breathing frequency (Figure 8c) decreases to $\Omega \sim \sqrt{3} \omega_{0}$ as $U$ is increased up to $U \sim 1$. At stronger interactions, the breathing frequency decreases further, unlike the continuum or small $k$ situations. This can be understood in the large $U$ (free fermion) limit: since the single-particle spectrum is dense for $k \sim 0.1$ (Figure 8 8 ), the difference between the $N$-th and $(N+2)$-th levels levels is smaller than $\sqrt{3} \omega_{0}$. There is an interesting plateau at $\Omega \sim \sqrt{3} \omega_{0}(U \sim 1)$ which is difficult to explain from single-particle considerations.

\section{SUMMARY AND DISCUSSION}

We have presented a detailed study of breathing modes for bosons on a tight-binding finite chain subject to a harmonic trap, and repulsively interacting through onsite interactions, as described by the 1D Bose-Hubbard model. We have used exact numerical diagonalization to treat up to 7 bosons, and used the excitation spectrum, overlaps after a quench, and explicit time evolution, to clarify various aspects of the problem. The continuum calculations of Ref. [10] are similar in spirit. In addition to connecting to the continuum limit, we have also highlighted deviations from this limit.

As in the continuum, the breathing mode frequency turns out to be the excitation energy of the lowest excited reflection-symmetric state. This is twice the trapping frequency, $2 \omega_{0}$, at small and large interactions, and it decreases to around $\sqrt{3} \omega_{0}$ at intermediate $U$. There are various lattice corrections to this continuum picture, which we have examined in the text, even for strong traps (Section $\mathrm{V})$.

Our results raise various open questions. Most prominent is the failure of the time-dependent Gutzwiller approximation to describe the breathing mode frequency at larger $U$ (Section IV). Since this is a popular method for time evolution of Bose-Hubbard systems [26, it is important to understand regimes and situations where it fails. Our results of Figure 7 should serve as a benchmark in further understanding of this issue.

It is possible to excite breathing modes through quenches of the on-site interaction $U$. While we expect essential features to be similar, it would be interesting to compare especially the overlap profiles analogous to Figures 5 for the trap quench.

We have restricted ourselves to low-density situations where Mott physics does not play a role. Clearly, dynamics in the presence of one or multiple Mott cores is an intriguing direction of study, especially if it is possible to go beyond Gutzwiller dynamics.
[1] D. S. Jin, J. R. Ensher, M. R. Matthews, C. E. Wieman, and E. A. Cornell, Phys. Rev. Lett. 77, 420
(1996). D. M. Stamper-Kurn, H.-J. Miesner, S. Inouye, 
M. R. Andrews, and W. Ketterle, Phys. Rev. Lett. 81, 500 (1998). F. Dalfovo, S. Giorgini, L. P. Pitaevskii, and S. Stringari, Rev. Mod. Phys. 71, 463 (1999). C. J. Pethick and H. Smith, Bose-Einstein Condensation in Dilute Gases, Cambridge University Press; 1st edition (2002); 2nd edition (2008).

[2] E. Haller, M. Gustavsson, M. J. Mark, J. G. Danzl, R. Hart, G. Pupillo, H. C. Nägerl, Science 325, 1224 (2009).

[3] M. Snoek, Phys. Rev. A 85, 013635 (2012).

[4] A. M. Rey, G. Pupillo, C. W. Clark, and C. J. Williams, Phys. Rev. A 72, 033616 (2005).

[5] E. Lundh, Phys. Rev. A 70, 033610 (2004); Phys. Rev. A 70, 061602(R) (2004).

[6] S. Peotta and M. Di Ventra, arXiv:1303.6916.

[7] G. Brandino, J.-S. Caux, and R. M. Konik, arXiv:1301.0308. J.-S. Caux and R. M. Konik, Phys. Rev. Lett. 109, 175301 (2012).

[8] S. Zöllner, H.-D. Meyer, and P. Schmelcher, Phys. Rev. A 75, 043608 (2007).

[9] I. Brouzos and P. Schmelcher, Phys. Rev. A 85, 033635 (2012).

[10] R. Schmitz, S. Krönke, L. Cao, and P. Schmelcher, arXiv:1306.5665.

[11] J. Grond, A. I. Streltsov, A. U. J. Lode, K. Sakmann, L. S. Cederbaum, and O. E. Alon, arXiv:1307.1667.

[12] G. E. Astrakharchik, J. Boronat, J. Casulleras, and S. Giorgini, Phys. Rev. Lett. 95, 190407 (2005).

[13] C. Menotti and S. Stringari, Phys. Rev. A 66, 043610 (2002).

[14] M. Kraemer, L. Pitaevskii, and S. Stringari, Phys. Rev. Lett. 88, 180404 (2002).

[15] T. Kimura, Phys. Rev. A 66, 013608 (2002).

[16] J. N. Fuchs, X. Leyronas, R. Combescot, Phys. Rev. A 68, 043610 (2003); Laser Physics 14,1 (2004).

[17] I. E. Mazets, Eur. Phys. J. D 65, 43 (2011).

[18] H. Moritz, T. Stöferle, M. Köhl, and T. Esslinger, Phys. Rev. Lett. 91, 250402 (2003).

[19] S. Stringari, Phys. Rev. A 58, 2385 (1998).

[20] T. Haugset and H. Haugerud, Phys. Rev. A 57, 3809 (1998).
[21] The function $p(n)$ generates the well-known sequence $1,1,2,3,5,7,11,15, \ldots$ counting the number of integer partitions of the integer $n$. This is listed as sequence A000041 in the Online Encyclopedia of Integer Sequences, http://oeis.org.

[22] The number of partitions of the integer $n$ into a maximum of $N$ parts, $p_{N}(n)$, differs from the unrestricted sequence $p(n)$ at larger $n$. For $N=2,3,4,5,6,7,8$, the sequences $p_{N}(n)$ are listed as A008619, A001399, A001400, A001401, A001402, A008636, A008637, in the Online Encyclopedia of Integer Sequences, http://oeis.org

[23] F. Deuretzbacher, K. Bongs, K. Sengstock, and D. Pfannkuche, Phys. Rev. A 75, 013614 (2007).

[24] L. Brey, N. F. Johnson, and B. I. Halperin, Phys. Rev. B 40, 10647 (1989). M. Bonitz, K. Balzer, and R. van Leeuwen, Phys. Rev. B 76, 045341 (2007).

[25] The Gutzwiller approximation is reviewed in Section 3.4 of: M. Lewenstein, A. Sanpera, V. Ahufinger, B. Damski, A. Sen (De), and U. Sen, Adv. Phys. 56, 243 (2007).

[26] D. S. Rokhsar and B. G. Kotliar, Phys. Rev. B 44, 10328 (1991). W. Krauth, M. Caffarel, and J.-P. Bouchaud, Phys. Rev. B 45, 3137 (1992). K. Sheshadri, H. R. Krishnamurthy, R. Pandit, and T. V. Ramakrishnan, Europhys. Lett. 22, 257 (1993). D. Jaksch, C. Bruder, J. I. Cirac, C. W. Gardiner, and P. Zoller, Phys. Rev. Lett. 81, 3108 (1998). D. Jaksch, V. Venturi, J. I. Cirac, C. J. Williams, and P. Zoller, Phys. Rev. Lett. 89, 040402 (2002). B. Damski, J. Zakrzewski, L. Santos, P. Zoller, and M. Lewenstein, Phys. Rev. Lett. 91, 080403 (2003). J. Zakrzewski, Phys. Rev. A 71, 043601 (2005). X. Lu and Y. Yu, Phys. Rev. A 74, 063615 (2006). M. Snoek and W. Hofstetter, Phys. Rev. A 76, 051603(R) (2007). C. Trefzger, C. Menotti, and M. Lewenstein, Phys. Rev. A 78, 043604 (2008). P. Navez and R. Schtzhold, Phys. Rev. A 82, 063603 (2010). S. Natu, K. Hazzard, and E. Mueller, Phys. Rev. Lett. 106, 125301 (2011).

[27] J. P. Ronzheimer, M. Schreiber, S. Braun, S. S. Hodgman, S. Langer, I. P. McCulloch, F. Heidrich-Meisner, I. Bloch, and U. Schneider, Phys. Rev. Lett. 110, 205301 (2013). 